\title{
$\mathrm{B} 3 \mathrm{G}$ 네트워크에서 동적 $\mathrm{QoS}$ 를 위한 핸드오버 연구
}

\author{
박상준 ${ }^{*}$, 이종찬 ${ }^{1}$ \\ ${ }^{1}$ 군산대학교 컴퓨터정보공학과
}

\section{A study of handover for dynamic QoS in B3G networks}

\author{
Sang Joon Park ${ }^{1^{*}}$ and Jong Chan Lee ${ }^{1}$ \\ ${ }^{1}$ Dept. of Computer Information Eng., Kunsan National University
}

\begin{abstract}
요 약 B3G 네트워크에서 서비스 제공자와 사용자 사이에 SLA (Service Level Agreement)가 정해지면 서비스 제공 자는 정해진 QoS 맞게 서비스를 제공하여야 한다. 하지만 네트워크는 상황은 시간에 따라 변하며, 서비스 제공 환경 이 변할 수 있다. 따라서 네트워크 서비스에 대해서 동적인 QoS 관리 방안이 고려되어야 한다. 본 논문에서는 B3G 융합망에서 네트워크 상황에 따라 동적인 $\mathrm{QoS}$ 를 위한 핸드오버 방안에 대해 제안한다. $\mathrm{B} 3 \mathrm{G}$ 네트워크에서 동적인 $\mathrm{QoS}$ 관리를 위하여 이종의 망을 이용하게 된다.
\end{abstract}

\begin{abstract}
The SLA (Service Level Agreement) is once determined between service provider and users in B3G networks so that the network service must be provided as QoS management. Here, the network situation is changed as the time flow, and the service environment is also altered. Hence, dynamic QoS management scheme should be considered. In this paper, we propose a handover scheme for dyanamic QoS as the network situation in B3G networks. The heterogeneous networks can be used for dynamic QoS management in B3G networks.
\end{abstract}

Key Words : B3G networks, SLA, Dynamic QoS, Handover, ISHO

\section{1. 서론}

네트워크 서비스는 기존의 단일한 서비스제공 방식에 서 벗어나 최근에는 이종의 네트워크에서 다양한 서비스 로의 확대를 요구하고 있다. $\mathrm{B} 3 \mathrm{G}$ (Beyond Three Generation) 네트워크는 새로운 하나의 네트워크 시스템 이 아니고 이러한 대규모의 이동통신시스템 및 이종의 서비스를 연동을 통하여 하나의 IP 기반 네트워크에서 사용하는 것처럼 자유로운 서비스를 제공하는 기술이다 [1-3]. B3G 네트워크에 대한 연구는 ITU-R WP 8F를 중 심으로 진행되고 있으며, 여러 나라에서도 ITU-R WP $8 \mathrm{~F}$ 와 더불어 연구를 진행하고 있다.

계층적으로 연결된 네트워크 사이에서 상호 연동을 위 한 자원관리는 $\mathrm{B} 3 \mathrm{G}$ 네트워크 개발에서 대단히 중요한 위치를 차지하며 QoS 레벨을 결정하는 요소가 될 것이다
$[5,6]$. 따라서 이러한 이종의 액세스 네트워크에 의하여 구성되는 $\mathrm{B} 3 \mathrm{G}$ 네트워크에서 seamless service, seamless 핸드오버 그리고 end-to-end QoS 관리에 대한 연구가 진 행되고 있다[4]. B3G 네트워크에서 이종의 네트워크 사 이에서 발생하는 핸드오버는 기존의 이동 단말기의 이동 에 의해 발생하는 핸드오버 이외에 서비스 특성에 따라 발생하는 핸드오버도 필요하게 되었다. 어떠한 상황에서 이종 네트워크 사이에서 핸드오버가 발생할 것인지 또한 이종 네트워크 간의 핸드오버에서 고려되어야 할 사항이 무엇인지에 대한 방안이 제공되어야 한다. 이종 네트워크 사이에서 발생하는 핸드오버에 대해서 고려해야 할 사항 으로 QoS 변동에 대한 mapping과 서비스 레벨에 대한 조 정 등이 있을 수 있다. End-to-end QoS 관리는 이종 네트 워크 환경에서 $\mathrm{QoS}$ 변동이 발생할 경우에 $\mathrm{B} 3 \mathrm{G}$ 네트워크 에서 대처해야 할 사항으로, 이동 가입자가 송수신하는

"교신저자 : 이종찬

접수일 11년 01월 28일 
트래픽에 대한 제어를 통하여 QoS 변동에 어떻게 대처해 야 하는지에 방안이 제시되어야 한다[7,8].

$\mathrm{B} 3 \mathrm{G}$ 네트워크에서 또한 고려해야 할 사항은 어떠한 구조를 통하여 이종 액세스 네트워크에 대한 연결 및 연 동을 제공할 것인가에 대해서다. 이종 액세스 네트워크의 연결을 위하여 네트워크의 구조에 설계와 연동에 필요한 기능들에 대한 연구가 진행되고 있으며, 이러한 연동 구 조에서 서비스 사용자에 대한 서비스 사용의 방안도 제 시되고 있다. 본 논문에서는 $\mathrm{B} 3 \mathrm{G}$ 네트워크에서 이종 액 세스 네트워크의 구성 그리고 연동을 위하여 필요한 사 항에 대해 기술한다. 또한 $\mathrm{B} 3 \mathrm{G}$ 네트워크에서의 동적 $\mathrm{QoS}$ 를 위한 이종 네트워크의 핸드오버에 대한 방안을 제 안하고, 시뮬레이션을 통하여 제안한 방안에 대한 성능 분석을 한다.

본 논문의 구성은 다음과 같다. 제 2장에서는 기존의 트래픽 제어 방식을 기술한다. 제 3장에서는 동적 $\mathrm{QoS}$ 를 위한 트래픽 제어 방식과 핸드오버 방안에 대해 기술하 며, 제안한 방식에 대한 성능 분석을 한다. 끝으로 4장에 서 결론을 맺는다.

\section{2. 트래픽 제어}

이동 단말기 사용자는 서비스 제공자와 $\mathrm{SLA}$ 를 맺고 통신 서비스를 사용하게 된다[10]. 협약된 통신 서비스를 기반으로 SLS (Service Level Specification) 파라미터에 의한 하위 서비스 파라미터가 정해지며 네트워크상에서 해당 파라미터의 수준을 유지하기 위하여 관리를 제공한 다. Diffserv 방식은 IP 기반의 트래픽 제어를 통하여 end-to-end QoS 관리를 위한 방안이다. Disffserv 방식에 의한 트래픽 제어는 서비스 특성에 따라 트래픽의 종류 를 분류하고 이에 대한 우선순위를 부여하여 관리하는 것이다. 따라서 SLA에 의하여 트래픽이 특성과 우선순위 가 결정되면 하위 트래픽 전송을 위한 TCA (Traffic Conditioning Agreements)가 결정된다. 먼저 Diffserv 방 식에서는 트래픽의 분류를 제공 서비스의 특성에 맞게 크게 $\mathrm{EF}$ (Expedited Forwarding), AF (Assured Forwarding), BE (Best Effort) 세 가지로 분류하였다.

- $\mathrm{EF}$ 트래픽 : $\mathrm{EF}$ 트래픽의 경우 패킷 전송에 대한 낮 은 손실율, 낮은 지연, 낮은 jitter등을 제공하며 보장 된 end-to-end 전송을 제공한다. 따라서 $\mathrm{EF}$ 트래픽 은 다른 여타의 트래픽 종류 보다 우선순위가 높으 며 트래픽 과부하 시에도 높은 우선순위가 유지되며 서비스가 지원된다. 따라서 $\mathrm{EF}$ 트래픽은 실시간 대 화형 서비스를 위하여 제공될 수 있다.
- AF 트래픽 : 최소한의 보장된 대역폭을 제공하는 서 비스로서 $\mathrm{EF}$ 트래픽 보다 우선순위가 낮은 트래픽 이다. IETF에서는 AF 트래픽에 대해 4개의 클래스 와 각 클래스별 세 가지 (low drop, medium drop, high drop)의 우선순위를 구별하였다. 트래픽 별 우 선순위는 네트워크상에서 트래픽 과부하가 발생 하 였을 경우 버퍼에서 우선순위에 의하여 트래픽을 폐 기하는 것을 의미한다. 트래픽의 과부하 시에 버퍼 의 각 패킷을 조사하여 하위의 패킷을 폐기하여 우 선순위가 높은 패킷에 대한 서비스를 높이는 것이 다. $\mathrm{AF}$ 트래픽의 경우 실시간 streaming 트래픽과 같은 서비스가 제공될 수 있다.

- $\mathrm{BE}$ 트래픽 : 기존의 IP 데이터 패킷 전송을 위한 것 으로 가장 우선순위가 낮은 것으로 트래픽 과부하 시에 전송에 대한 보장을 제공하지 못하는 트래픽이다.

그림 3은 IETF에서 제시한 Diffserv의 트래픽 조절 기 능에 대한 일반적인 형태를 보이고 있다.

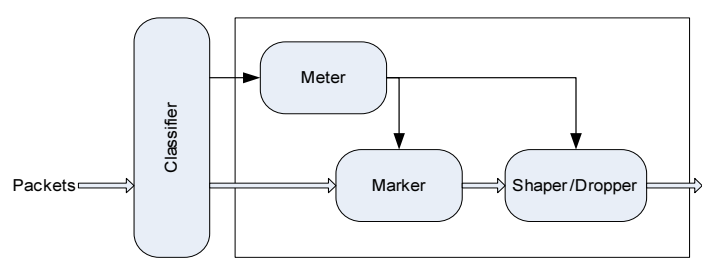

[그림 1] 트래픽 제어

\section{3. 동적 QoS 관리}

\section{1 동적 트래픽 관리}

본 논문에서는 SLA의 서비스 다양화를 위하여 $\mathrm{QoS}$ 변동을 위한 Diffserv 방식을 고려한다. 동적 QoS 변동을 위한 Diffserv 방식은 버스트한 트래픽 전송으로 트래픽 의 부하가 높아질 경우 트래픽 조절 부분에서 동적으로 트래픽 클래스를 관리하는 것이다. 통신 서비스를 위하여 이동 단말기에서 전송되는 IP 트래픽의 클래스를 $\mathrm{EF}, \mathrm{AF}$ (High, Medium, Low), BE 크게 다섯 가지로 분류한다. $\mathrm{AF}$ 클래스는 세 개의 우선순위로 구분하며 트래픽의 부 하가 높아질 때에 패킷 폐기에 반영한다. $\mathrm{AF}$ 트래픽 클 래스에 대한 서비스 우선순위는 (AF_High > AF_Medium > AF_Low) 순이다. 패킷이 Diffserv 트래픽 조절 부분에 도착하면 협약된 서비스에 따라 각 트래픽 클래스는 분류되어 전송된다. 일단 통신 서비스를 위한 네트워크 트래픽이 발생하면 동일 망이든지 혹은 이기종 
망이든지 네트워크의 트래픽 조절을 하는 것이다. 본 논 문에서는 각 트래픽 레벨에 대한 Diffserv 트래픽 전송 제어 를 위하여 WFQ (Weighted Fair Queueing) 스케줄링 방식을 사용 한다[9]. WFQ 방식은 각 서비스 클래스 별 서비스 가중치를 통하여 패킷을 전송하는 서비스이다. Diffserv 트래픽 관리에서 제어하는 서비스 클래스의 수가 $n$ 이라 고 가정하고, 특정 트래픽 클래스를 $i$ 이면 $i=1,2,3, \ldots, n$ 이다. 만일 $\Psi_{i}$ 을 특정 서비스 $i$ 의 weighted factor이라 고 한다면 서비스 클래스 $i$ 의 대역폭은 다음과 같다.

$$
\Delta B_{i}=\frac{\Psi_{i}}{\sum_{k=1}^{n} \Psi_{k}} B_{A} .
$$

여기서 $B_{A}$ 는 전체 대역폭을 나타내며, $\Psi_{i}>0$ 이다. 만일 시간 $t$ 에서 특정 서비스 클래스 $i$ 의 $m$ 번째 패 킷에 대한 timestamp $\left(T_{i}^{m}\right)$ 는 다음과 같다. 서비스 클래 스 $i$ 의 $m$ 번째 패킷의 timestamp는 $m-1$ 번째 패킷이 전송되고 바로 뒤이은 시점부터 패킷의 길이만큼이다.

$$
T_{i}^{m}=\max \left[T_{i}^{m-1}, V(t)\right]+\frac{L_{i}^{m}}{r_{i}} .
$$

여기서 $V(t)$ 는 가상의 시간함수이며, $L_{i}^{m}$ 은 서비스 클래스 $i$ 의 $m$ 번째 패킷의 길이이고, $r_{i}$ 는 서비스 클래 스 $i$ 의 패킷 전송률이다.

\section{2 동적 서비스 기반 핸드오버}

본 논문에서는 동적 $\mathrm{QoS}$ 관리를 위하여 서비스 품질 의 상승 시에 ISHO (Inter-system Handover) 관리를 위한 방안을 제안한다. 기존의 이동 단말기의 이동에 의한 ISHO가 아닌 end-to-end QoS 상승을 위하여 수행하는 ISHO이다. 이동 단말기 사용자가 세션을 open하거나 통 신 서비스를 이용하는 중에 다른 중첩된 이기종 네트워 크로 $\mathrm{QoS}$ 상승을 위하여 $\mathrm{ISHO}$ 를 발생한다. UMTS (Universal Mobile Telecommunication System)와 WLAN (Wireless LAN)의 경우 UMTS 시스템 서비스를 이용하 는 중간에 WLAN 시스템으로 QoS 상승을 위한 ISHO를 수행할 수 있다. 이 경우 UMTS 네트워크와 WLAN이 서 로 중첩된 구조를 이루고 있고 중첩 지역 안에 사용자 있 을 경우이다. 또한 이러한 $\mathrm{ISHO}$ 를 위하여 WLAN의 경우 UMTS 시스템의 통신 서비스 클래스 보다 더 나은 트래 픽 전송 환경을 제공하여야 한다.

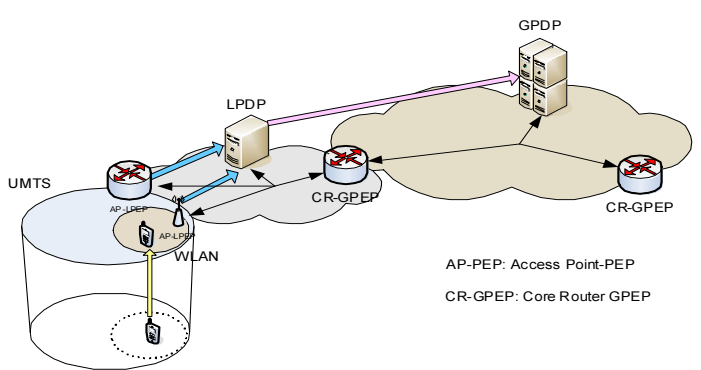

[그림 2] 동적 $\mathrm{QoS}$ 관리구조

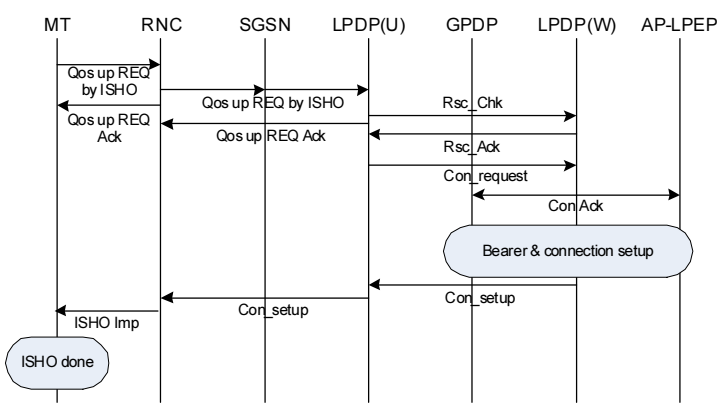

[그림 3] 동적 $\mathrm{QoS}$ 를 위한 시그널링

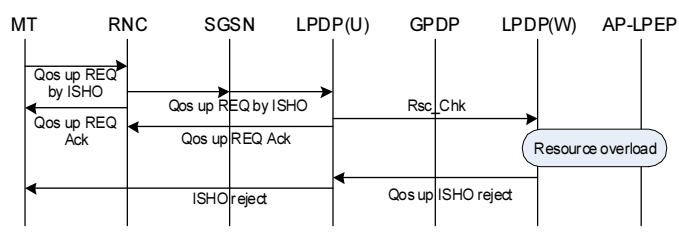

[그림 4] 핸드오버 거부 시그널링

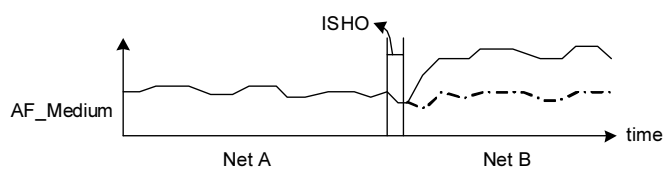

(a)

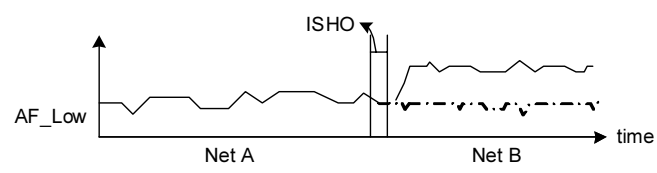

(b)

[그림 5] 핸드오버에 따른 트래픽 제어

WLAN 시스템에서 심각한 트래픽 과부하로 인하여 정상적인 서비스가 힘들 경우 UMTS로의 서비스 전환 또 한 $\mathrm{QoS}$ 상승을 위한 $\mathrm{ISHO}$ 라고 하겠다. 그림 2은 UMTS 네트워크에서 이동 단말기가 QoS 상승을 위하여 WLAN 으로 $\mathrm{ISHO}$ 를 수행하는 것을 보이고 있다. QoS 상승을 
위한 ISHO 서비스는 SLA의 체결에 의해 제공될 수 있으 며 이동 단말기가 UMTS 지역에 있다가 WLAN 지역으 로 진입하였을 시에 수행될 수 있으며, 단말기가 UMTS 와 WLAN 중첩 지역에서 서비스를 사용하다가 서비스 레벨을 올리기 위하여 ISHO를 수행하는 경우로 나누어 질 수 있다. 이동 단말기는 UMTS 네트워크에 있다가 WLAN hot spot 지역에 진입할 경우 혹은 WLAN광의 중 첩 지역에 있을 경우 $\mathrm{QoS}$ 상승을 위한 ISHO 요구 메시 지를 $\mathrm{RNC}$ 에게 전달한다. $\mathrm{QoS}$ 상승을 위한 ISHO 요구 메시지를 받은 RNC는 UMTS 관리 LPDP (Local Policy Decision Point)에게 요구 메시지를 전달한다. 요구 메시 지를 전달받은 LPDP는 GPDP (Global Policy Decision Point)를 통하여 WLAN의 LPDP에게 QoS 상승을 위한 자원 상태를 점검하고 $\mathrm{QoS}$ 상승이 가능하면 이를 UMTS $\mathrm{LPDP}$ 에게 알리며 이동 단말기의 정보를 통하여 $\mathrm{ISHO}$ 를 위한 연결 설정 및 자원할당을 한다. 연결 설정 후에 이 동 단말기에 대해 QoS 상승을 위한 ISHO 수행을 전달한 다. 만일 WLAN에서의 트래픽 과부하로 인하여 QoS 상 승이 여의치 않을 경우 WLAN의 LPDP는 QoS 상승을 위한 이동 단말기의 ISHO 요구를 거절한다 그림 4. WLAN의 LPDP는 자원점유지표와 이동 단말기의 외부 프로파일을 참조하여 해당 네트워크의 자원 상태를 확인 한 후에 최종 ISHO 거부 판단을 하고 결과를 이동 단말 기에게 전달한다. WLAN에서 자원 소모에 의한 ISHO 거 부는 일정한 WLAN에서 제공할 수 있는 트래픽 전송율 을 기준하여 현재 제공되고 있는 트래픽 서비스를 기반 으로 요청 서비스에 대한 $\mathrm{QoS}$ 제공이 가능한지에 대한 결정에 의한 것이다. QoS 상승은 Diffserv 버퍼의 부하와 는 상관없이 수행할 수 있으며 단말기는 ISHO를 통하여
동적 트래픽 관리 방식과 유사하게 트래픽 클래스의 레 벨을 올리는 것이다. QoS 상승을 위한 ISHO는 이기종 네트워크에서 통신 서비스에 대해 상승된 $\mathrm{QoS}$ 를 제공할 수 있어야 한다. 그림 5 는 이동 단말기 호에 대해 QoS 상 승을 위하여 ISHO 수행 후에 QoS 변동을 보이고 있다. 그림 5(a)는 AF_Medium 트래픽 클래스를 사용하는 이동 단말기가 QoS 상승을 위하여 $\mathrm{ISHO}$ 를 수행하는 것을 보 이고 있다. 이러한 QoS 상승을 위한 ISHO 수행은 버퍼 임계치의 부하와는 상관없이 서비스 제공자와의 SLA에 의하여 핸드오버를 할 수 있다. 그림에서 보면 중첩된 네 트워크 Net A에서 이동 단말기가 네트워크 Net B로 $\mathrm{ISHO}$ 를 수행하는 것을 보이고 있다. ISHO를 수행하지 않는다면 그림에서처럼 트래픽 $\mathrm{QoS}$ 는 점선으로 보이는 것과 같이 여전히 동일한 레벨을 보이며, ISHO 수행한다 면 $\mathrm{QoS}$ 상승을 보이고 있다. 그림 5(b)는 AF_Low 트래 픽 클래스에 대한 $\mathrm{ISHO}$ 를 보이고 있다. 이동 단말기는 ISHO 이후에 상승된 end-to-end $\mathrm{QoS}$ 를 받을 수 있다. $\mathrm{QoS}$ 상승을 위한 ISHO는 대상 네트워크에서 이전의 네 트워크 통신 서비스 클래스 보다 더 나은 트래픽 $\mathrm{QoS}$ 를 보장하여야 한다. UMTS와 WLAN의 경우 UMTS에 이동 하던 단말기가 WLAN 중첩 지역에서 WLAN으로 ISHO 를 수행할 수 있는 것이다. WLAN으로 ISHO를 수행함으 로이동 단말기는 UMTS 시스템에서 보다 더 나은 QoS 받을 수 있다. QoS 상승을 위한 ISHO는 Diffserv 버퍼의 임계치와 상관없이 수행할 수 있으며 SLA에 의해서 제 공될 수 있다.버퍼 임계치 부하에 따른 상황을 고려하여 동적 트래픽 제어 방식과 함께 $\mathrm{QoS}$ 상승을 위한 $\mathrm{ISHO}$ 를 고려한다면 다음 식과 같이 나타낸다.

$$
\begin{aligned}
& \Delta B_{A F_{-} \text {Meidum }}=\sum_{\gamma^{\prime}=\gamma^{\prime} 1}^{A F M_{\gamma^{\prime}}} \Delta B_{\gamma^{\prime}}-\sum_{Q H-\gamma^{\prime}=\gamma_{q l^{\prime}}}^{A F M_{O H-\gamma^{\prime}}} \Delta B_{Q H-\gamma^{\prime}}+\sum_{\gamma^{\prime \prime}=\gamma^{\prime \prime} 1}^{A F M_{\gamma^{\prime \prime}}} \Delta B_{\gamma^{\prime \prime}} \\
& = \begin{cases}\sum_{\gamma^{\prime}=\gamma^{\prime} 1}^{A F M_{\gamma^{\prime}}} \frac{\Psi_{\gamma^{\prime}}}{\sum \Psi} B_{A} & \text { if } T_{b} \leq M t \\
B_{A}\left[\sum_{\gamma^{\prime}=\gamma^{\prime} 1}^{A F M_{\gamma^{\prime}}} \frac{\Psi_{\gamma^{\prime}}}{\sum \Psi}-\sum_{Q H-\gamma^{\prime}=\gamma^{\prime} l^{\prime}}^{A F M_{O H-\gamma^{\prime}}} \frac{\Psi_{Q H-\gamma^{\prime}}}{\sum \Psi}+\sum_{\gamma^{\prime \prime}=\gamma^{\prime \prime 1}}^{A F M_{\gamma^{\prime \prime}}} \frac{\Psi_{\gamma^{\prime \prime}}}{\sum \Psi}\right] & \text { if } T_{b}>M t\end{cases} \\
& \Delta B_{A F_{-} L o w}=\sum_{\chi^{\prime}=\chi^{\prime} 1}^{A F L_{\chi^{\prime}}} \Delta B_{\chi^{\prime}}-\sum_{Q H-\chi^{\prime}=\chi_{q^{\prime} 1}}^{A F L_{Q H-\chi^{\prime}}} \Delta B_{Q L-\chi^{\prime}}+\sum_{\chi^{\prime \prime}=\chi^{\prime \prime 1}}^{A F L_{\chi^{\prime \prime}}} \Delta B_{\chi^{\prime \prime}} \\
& = \begin{cases}\sum_{\chi^{\prime}=\chi^{\prime} 1}^{A F L_{\chi^{\prime}}} \frac{\Psi_{\chi^{\prime}} \Psi_{A}}{B^{\prime}} & \text { if } T_{b} \leq L t \\
B_{A}\left[\sum_{\chi^{\prime}=\chi^{\prime} 1}^{A F L_{\chi^{\prime}}} \frac{\Psi_{\chi^{\prime}}}{\sum \Psi}-\sum_{Q H-\chi^{\prime}=\chi_{q^{\prime} l^{1}}}^{A F L_{O H-\chi^{\prime}}} \frac{\Psi_{Q H-\chi^{\prime}}}{\sum \Psi}+\sum_{\chi^{\prime \prime}=\chi^{\prime \prime 1}}^{A F L_{\chi^{\prime \prime}}} \frac{\Psi_{\chi^{\prime \prime}}}{\sum \Psi}\right] & \text { if } T_{b}>L t\end{cases}
\end{aligned}
$$




$$
\begin{aligned}
& \sum_{\gamma^{\prime}=\gamma^{\prime} 1}^{A F M_{\gamma^{\prime}}} \Delta B_{\gamma^{\prime}\left(t_{\delta-1}, t_{\delta}\right)}-\sum_{Q H-\gamma^{\prime}=\gamma^{\prime} h^{\prime} 1}^{A F M_{Q H-\gamma^{\prime}}} \Delta B_{Q H-\gamma^{\prime}\left(t_{\delta-1}, t_{\delta}\right)}+\sum_{\gamma^{\prime \prime}=\gamma^{\prime \prime} 1}^{A F M_{\gamma^{\prime \prime}}} \Delta B_{\gamma^{\prime \prime}\left(t_{\delta-1}, t_{\delta}\right)}= \\
& \Delta B_{\gamma^{\prime} 1\left(t_{\delta-1}, t_{\delta}\right)}+\Delta B_{\gamma^{\prime} 2\left(t_{\delta-1}, t_{\delta}\right)}+\Delta B_{\gamma^{\prime} 3\left(t_{\delta-1}, t_{\delta}\right)}+\cdots+\Delta B_{A F M_{\gamma^{\prime}}\left(t_{\delta-1}, t_{\delta}\right)} \\
& -\Delta B_{\gamma^{\prime}{ }^{\prime} 1\left(t_{\delta-1}, t_{\delta}\right)}-\Delta B_{\gamma^{\prime}{ }_{q h} 2\left(t_{\delta-1}, t_{\delta}\right)}-\Delta B_{\gamma^{\prime}{ }_{q h} 3\left(t_{\delta-1}, t_{\delta}\right)} \cdots-\Delta B_{A F M_{Q H-\gamma^{\prime}}\left(t_{\delta-1}, t_{\delta}\right)} \\
& +\Delta B_{\gamma^{\prime \prime} 1\left(t_{\delta-1}, t_{\delta}\right)}+\Delta B_{\gamma^{\prime \prime 2} 2\left(t_{\delta-1}, t_{\delta}\right)}+\Delta B_{\gamma^{\prime \prime} 3\left(t_{\delta-1}, t_{\delta}\right)}+\cdots+\Delta B_{A F M_{\gamma^{\prime \prime}}\left(t_{\delta-1}, t_{\delta}\right)} \\
& \begin{cases}\frac{B_{A}\left[t_{\delta-1}-t_{\delta}\right]\left(\Psi_{\gamma^{\prime} 1}+\Psi_{\gamma^{\prime} 2}+\Psi_{\gamma^{\prime} 3}+\cdots+\Psi_{A F M_{\gamma^{\prime}}}\right)}{\sum \Psi} & \text { if } T_{b} \leq M t \\
\frac{B_{A}\left[t_{\delta-1}-t_{\delta}\right]\left(\Psi_{\gamma^{\prime} 1}+\Psi_{\gamma^{\prime} 2}+\Psi_{\gamma^{\prime} 3}+\cdots+\Psi_{A F M_{\gamma^{\prime}}}\right)}{\sum \Psi} & \\
-\frac{B_{A}\left[t_{\delta-1}-t_{\delta}\right]\left(\Psi_{\gamma_{q h}^{\prime} 1}+\Psi_{\gamma_{q h}^{\prime} 2}+\Psi_{\gamma_{q h}^{\prime} 3}+\cdots+\Psi_{A F M_{Q H-\gamma^{\prime}}}\right)}{\sum \Psi^{\prime}} & \text { if } T_{b}>M t \\
+\frac{B_{A}\left[t_{\delta-1}-t_{\delta}\right]\left(\Psi_{\gamma^{\prime \prime} 1}+\Psi_{\gamma^{\prime \prime 2}}+\Psi_{\gamma^{\prime \prime} 3}+\cdots+\Psi_{A F M_{\gamma^{\prime \prime}}}\right)}{\sum \Psi} & \end{cases}
\end{aligned}
$$$$
\sum_{\chi^{\prime}=\chi^{\prime} 1}^{A F L_{\chi^{\prime}}} \Delta B_{\chi^{\prime}\left(t_{\delta-1}, t_{\delta}\right)}-\sum_{Q H-\chi^{\prime}=\chi_{q 1}^{\prime} 1}^{A F B_{O H-\chi^{\prime}}} \Delta B_{Q H-\chi^{\prime}\left(t_{\delta-1}, t_{\delta}\right)}+\sum_{\chi^{\prime \prime}=\chi^{\prime \prime} 1}^{A F L_{\chi^{\prime \prime}}} \Delta B_{\chi^{\prime \prime}\left(t_{\delta-1}, t_{\delta}\right)}=
$$

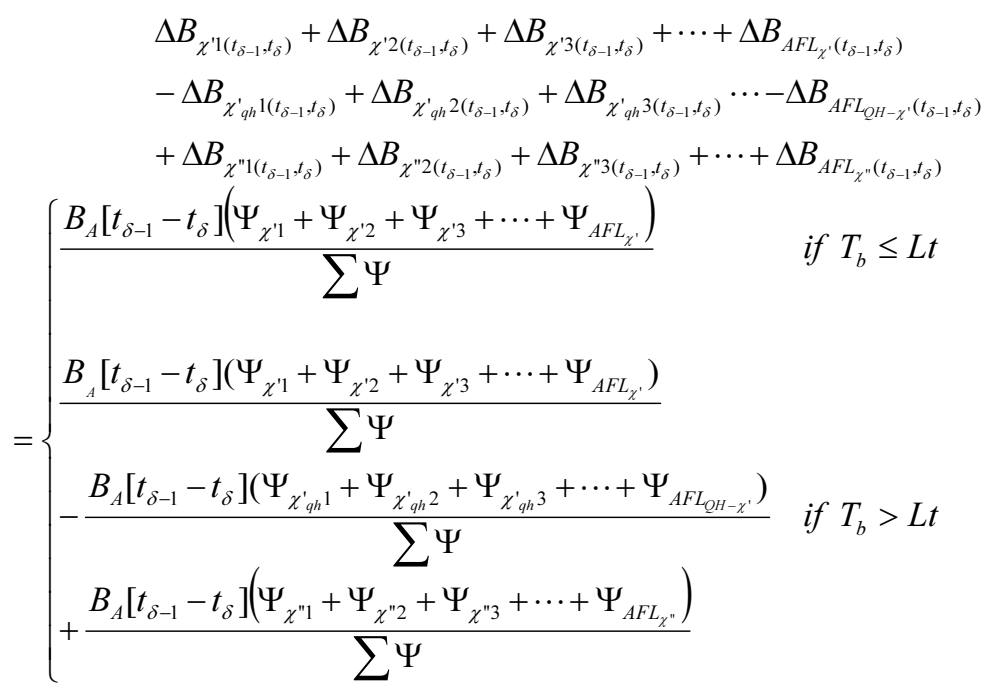

버퍼의 임계치를 고려하고, 시간 구간 $\left[t_{\delta-1}, t_{\delta}\right]$ 에서 AF_Medium과 AF_Low 클래스 서비스를 사용하는 각각 의 사용자에 대한 ISHO 대역폭 변동은 다음 식과 같이 나타낸다. 위의 식을 이용하여 QoS 상승을 위한 ISHO 수행에 대한 특정 사용자의 통신 서비스 (AF_Medium, $\mathrm{AF} \_$Low)의 전체 트래픽 전송량은 다음 식과 같다.

$$
\begin{gathered}
\Delta B_{A F_{-} \operatorname{Medium}(u)}\left[t_{s}-t_{0}\right]=\sum_{t=t_{0}}^{t_{h}} \frac{\Psi_{A F m(u)} B_{A}}{\sum \Psi}+\sum_{t=t_{h}}^{t_{s}} \frac{\Psi_{A F m\left(u_{q h}\right)} B_{A}}{\sum \Psi} \\
\Delta B_{A F_{-} \operatorname{Low}(u)}\left[t_{s}-t_{0}\right]=\sum_{t=t_{0}}^{t_{h}} \frac{\Psi_{A F w(u)} B_{A}}{\sum \Psi}+\sum_{t=t_{h}}^{t_{s}} \frac{\Psi_{A F w\left(u_{q h}\right)} B_{A}}{\sum \Psi}
\end{gathered}
$$


표 1과 표 2는 성능분석을 위한 시스템 파라미터를 나 타낸다.

[표 1] UMTS 트래픽 파라미터

\begin{tabular}{|c|c|}
\hline 트래픽 클래스 & 전송률 \\
\hline EF & $384-192 \mathrm{kbps}$ \\
\hline AF_High & $192-64 \mathrm{kbps}$ \\
\hline AF_Medium & $64-32 \mathrm{kbps}$ \\
\hline AF_Low & $32-16 \mathrm{kbps}$ \\
\hline $\mathrm{BE}$ & Best effort \\
\hline
\end{tabular}

[표 2] WLAN 트래픽 파라미터

\begin{tabular}{|c|c|}
\hline 트래픽 클래스 & 전송률 \\
\hline EF & $1 \mathrm{Mbps}-512 \mathrm{kbps}$ \\
\hline AF_High & $384-192 \mathrm{kbps}$ \\
\hline AF_Medium & $192-64 \mathrm{kbps}$ \\
\hline AF_Low & $64-32 \mathrm{kbps}$ \\
\hline $\mathrm{BE}$ & Best effort \\
\hline
\end{tabular}

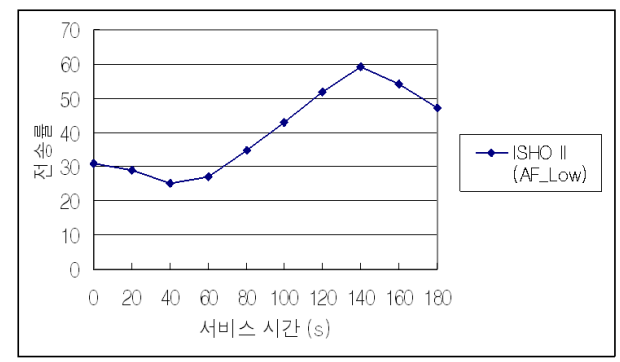

[그림 6] AF_Low 트래픽 변화

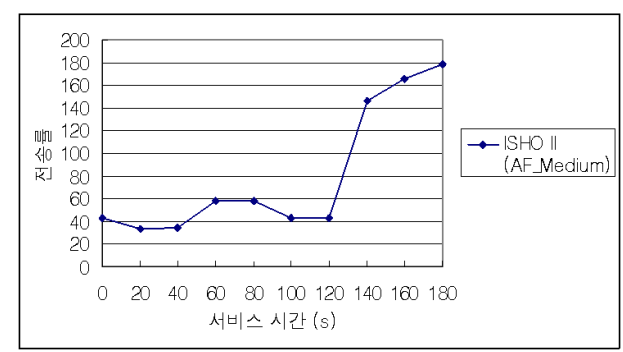

[그림 7] AF_Medium 트래픽 변화

그림 6은 UMST에서 AF_Low 클래스를 사용하는 단 말기가 중첩 지역에서 $\mathrm{ISHO}$ 를 수행하여 WLAN으로 서 비스 변경한 것을 보이고 있다. 그림 6의 경우 동적 $\mathrm{QoS}$
관리를 통하여 서비스 전송을 $64 \mathrm{kbps}$ 레벨로 상향 조정 한 것을 보이고 있다. 그림 7은 AF_Medium 클래스를 사 용하는 단말기가 중첩지역에서 $\mathrm{ISHO}$ 를 통하여 WLAN 서비스를 이용하는 것을 보이고 있다. 그림7은 $\mathrm{ISHO}$ 를 통하여 $64 \mathrm{kbps}$ 트래픽 레벨에서 $192 \mathrm{kbps}$ 로 서비스를 상향 조정한 것을 보이고 있다.

\section{4. 결론}

$\mathrm{B} 3 \mathrm{G}$ 네트워크로의 확장은 네트워크 자체의 확장뿐만 아니라 네트워크 서비스 확장도 의미한다. 네트워크 서비 스 확장은 단순한 여러 서비스의 확장에 제한할 수 있으 나 향후 네트워크 동적인 서비스 관리 측면에서 확대된 네트워크 서비스를 기대한다. 네트워크 서비스의 확장은 기술적인 부분뿐만 아니라 서비스 정책에 맞추어 제공될 수 있다. 따라서 이러한 정책적 서비스 확대를 고려한 기 술적인 서비스 제공을 고려해야 한다. 본 논문에서는 융 합망에서 동적인 $\mathrm{QoS}$ 관리를 위한 방안을 제안하였다. 이종의 네트워크에서 발생할 수 있는 $\mathrm{ISHO}$ 를 이용하여 동적 $\mathrm{QoS}$ 를 제공한다. 따라서 서비스 사용자에게 네트워 크 상황에 따라 SLA에 근거한 서비스 확대가 가능할 수 있다. 따라서 단일 네트워크에서 기대하지 못하였던 동적 서비스 관리가 융합망에서는 적용될 수 있을 것으로 기 대된다.

\section{참고문헌}

[1] Werner mohr and Walter Konhauser, "Access Network Evolution Beyond Third Generation Mobile Communications ,"IEEE Communication Magazine, pp. 122 - 133, December 2000.

[2] Mahbubul Alam, Ramjee Prasad and John R. Farserotu, "Quality of Service among IP-Based Heterogeneous Networks," IEEE Personal communications, pp. 18-24, December 2001.

[3] Theodore B. Zahariadis, Konstantinos G. Vaxevanakis, Christos P. Tsantilas, Nikolaos A. Zervos and Nikos A. Nikolaou, "Global Roaming in Next-Generation Networks," IEEE Communications Magazine, pp. 145-151, February 2002.

[4] Gang Wu, Mitsuhiko Mizuno and Paul J.M. Havinga, "MIRAI Architecture for Heterogeneous Network," "IEEE Communications Magazine, pp. 126-134, February 2002. 
[5] Giovanni Cortese, Roberto Fiutem, Piergiorgio Cremonese, Salvatore D'Antonio,Marcello Esposito, Simon Pietro Romano and Ada Diaconescu, "End-User Services in Premium IP Networks," IEEE Communications Magazine, pp. 54-60, January 2003.

[6] Victor Marzues, Rui L. Aguiar, Carlos Garcis, Jose Ignacio Moreno, Christophe Beaujean, Eric Melin and Marco Liebsch, "An IP-Based QoS Architecture for 4G Operator Scenarios," IEEE Wireless Communications, pp. 54-62, June 2003.

[7] Suk Yu Hui and Kai Hau Yeung, "Challenges in the Migration to 4G Mobile Systems,'IEEE Communications Magazine, pp. 54-59, December 2003.

[8] Louis C. Schreier and Michael B. Davis, "System-Level Resource Management for Network-Based Multimedia Applications," Proceedings of the 5th International Workshop on Network and Operation System Support for Digital Audio and Video, pp. 121-124, 1995.

[9] Francis-Cobley, P. and Davies, N., "Performance Implications of QoS Mapping in Heterogeneous Networks involving ATM,'IEEE ICATM-98, pp. 529-535, June 1998.

[10] Wei Zhuang, Yung-Sze Gan, Kok-Jeng Loh, and Kee-Chaing Chua, "Policy-Based QoS Management Architecture in an Integrated UMTS and WLAN Environment," Communication Magazine, IEEE, vol.41, November 2003.

\section{박 상 준(Sang Joon Park)}

[정회원]

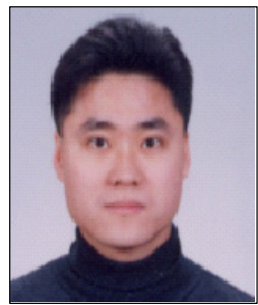

- 1998년 : 숭실대학교 컴퓨터학과 석사

- 2002년 : 숭실대학교 컴퓨터학과 박사

- 2002년 2003년 : 런던대 ISG 박사후 과정

- 2004년 2007년 : 숭실대학교 정보미디어기술연구소 연구교수

- 2007년 현재 : 국립군산대학교 컴퓨터정보공학과 조 교수

<관심분야>

$\mathrm{B} 3 \mathrm{G}$, 센서 네트워크, 인터넷 망 분석, 디지털포렌식

\section{이 종 찬(Jong Chan Lee)}

[정회원]

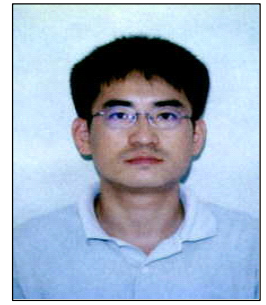

- 1996년 : 숭실대학교 대학원 전 자계산학과 석사

- 2000년 : 숭실대학교 대학원 컴 퓨터과학과 박사

- 2000년 2005년 : ETRI 선임연 구원

- 2005년 현재 : 국립군산대학 교 컴퓨터정보공학과 부교수

<관심분야>

이동통신, 센서 네트워크, 디지털포렌식 